\title{
Quantum Dot Cellular Automato Process in Image Processing Techniques
}

\author{
M. Subhashini \\ Senior Executive, Lacustrine Technologies Pvt. Ltd., Chennai
}

\begin{abstract}
This paper presents the important of employing Quantum Dot Cellular Automato in Image processing. Quantum Dot Cellular Automato is anupcoming technology of Nano electronics ensuring very low power consumption, maximized clock speed, low silicon area utilization and performance enriching parallel processing techniques. Here we discussed the fundamental of image processing techniques and application role of quantum dot cellular automato in it.
\end{abstract}

Keywords - Quantum Dot Cellular Automato; Image Processing; Nano Electronics

\section{Introduction}

Digital image processing is a two-dimensional light intensity $f(x, y)$ value of $f$ at spatial co-ordinates $x$ and $y$. Color image processing employs colors which simplifies object identification and extraction. Human eye can discern nearly thousands of color shades. Three quantities for quality description includes radiance, luminance, brightness where radiance describes total energy from light source in watts, luminance describes amount of energy observed by observer from light source and brightness describes color sensation. The primary colors are blue, green and red. These primary colors added up to produce secondary colors. Magenta produced from the combination of red and blue where cyan is the combination of green and blue, yellow is the combination of red and green and white is the combination of all the three primary colors. The color models facilitate specification of colors in a standard 3-D co-ordinate system and subspace where each color represented as dots (single point). There are three color models they are represented in below table.

Table 1: Color models and their applications

\begin{tabular}{|l|l|}
\hline COLOR MODELS & APPLICATONS \\
\hline RGB & $\begin{array}{l}\text { Color monitors, color video } \\
\text { camera }\end{array}$ \\
\hline CMY & Color printer \\
\hline $\begin{array}{l}\text { YIQ (where I, Q are } \\
\text { chromatic components such } \\
\text { as in phase and Quadrature) }\end{array}$ & Color Tv broadcast \\
\hline
\end{tabular}

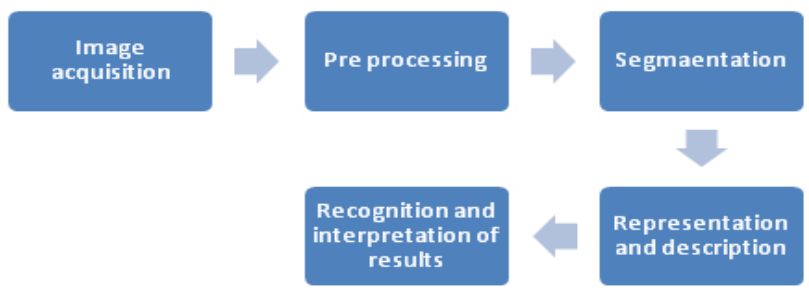

Fig. 1: Flow chart of digital image processing
The above flow chart explains steps in digital image processing. Image enhancement is defined as aggregate of pixels composing an image either in spatial or frequency domain where in spatial domain direct manipulation of pixel is taken place in case of frequency domain modification of Fourier transform of image will take place followed by convolution. Image enhancement techniques are discussed in figure 2 .

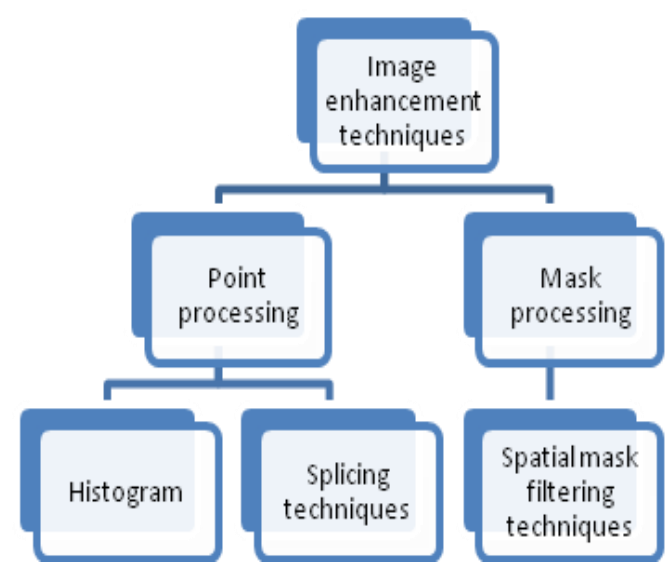

Fig.2: Image enhancement techniques

Point processing modifies grey level of pixel independently of neighbor whereas mask processing modifies each pixel in image to be enhanced. Point processing includes contract stretching technique to improve low contrast images explained in figure 3, compression of dynamic ranges when it exceeds the capability of display device. Gray level splicing is to highlight specific range of grey level. Example: Flaws in $\mathrm{x}-$ ray images, masses of water in satellite images. Bit plane splicing instead of highlighting intensity range highlights specific bit and Histogram processing denotes the probability of occurrence of grey level which gives us useful information about possibility of contrast enhancement and it is further classified as histogram equalization and histogram specification. 


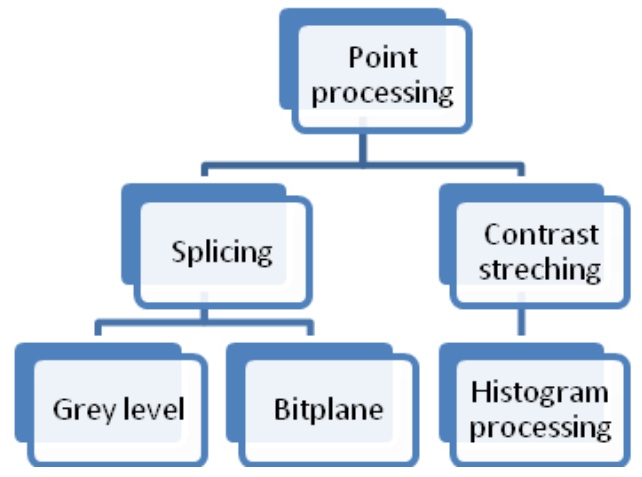

Fig. 3: Point processing techniques.

There are different types of spatial filters used in image enhancement techniques. The techniques are discussed in figure 4.

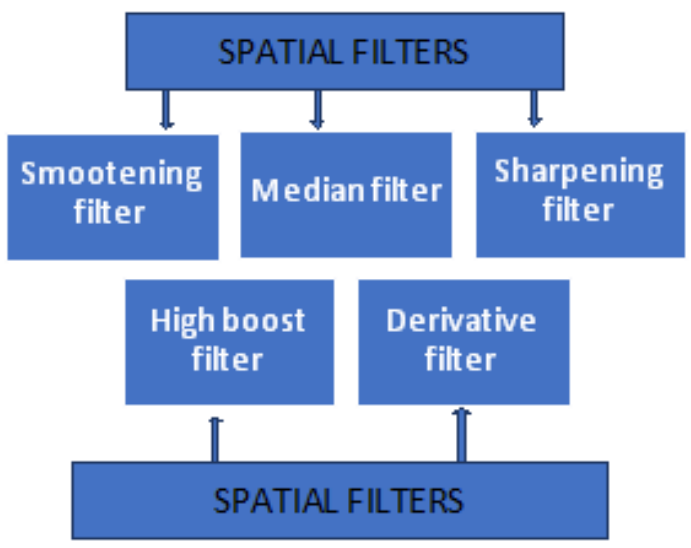

Fig. 4: Types of spatial filters

Smoothening filters used for blurring and noise reduction in pre-processing steps and removes small details from image. Median filters are used in noise reduction instead of blurring a grey level of pixel replaces median of grey level in neighbor of pixel. It preserves edge sharpness. Sharpening filters are used to highlight fine details and enhance blurred detail as a part of image acquisition. Image restoration involves inverse filtering and Least Mean Square (LMS) filter also called as Weiner filter, Image segmentation involves edge detection by Laplacian filter and uniform cost algorithm of Dijkstra. Thresholding process involves Global thresholding and Local thresholding methods. Gray level interpolation is classified as zero order interpolation and cubic convolution. Derivative filters make use of Robert cross gradient operator, prewitt operator and sobel operator.

\subsection{Image Classification}

The digital image classification is an area under the umbrella of image processing and basically dealt with pixel grouping in a raster image with the purpose for information extraction, after raster image is processed by applying image classification techniques, the resultant raster can be employed for the creation of thematic maps. These would further be analyzed or processed with suitable technique so that the final goal of image processing is met. The image classification techniques are classified in to three types depending on interaction between operator and the computer,

- Supervised image classification

- Un supervised image classification

- Object oriented image analysis

\section{Nano Electronics}

Electronic device today reduced to Nano scale size. Most image and video processing algorithms resulted in demand of speed and computational power. The Very Large-Scale Integration technologies includes silicon Nano wires, carbon Nano tubes, single electron transistors, resonant tunneling diode and single molecule device replaces conventional CMOS technology. Quantum Dot Cellular Automato (QCA) resulted in parallel pipelined architecture and speedy operation using electric charges position for coding information instead electric current flow and resulted in reduced power consumption and computational complexity. In today's age of digitalization, the presence of digital images and videos is almost omnipotent. Various quality images subjected to different level of noise parameters such as unrestricted motion of the camera on recording image, uncontrollable conditions of indoor and outdoor environment and susceptibility of noise while transmission. Image processing is done for purpose of noise reduction, Feature extraction, face recognition, skeletonizing, segmentation, thinning, thickening, shape extraction, object detection and tracking. Mathematical morphology is a less complex way of carrying out the image processing. VLSI implementation in image processing includes Implementation of nonlinear filters and video noise reduction.

\section{Quantum Dot Cellular Automato Process}

Cellular automata are the process for simplified mathematical modelling of complex structures which when implemented using coupled quantum dots is known as Quantum Dot Cellular Automata. It is a design of complex architecture in Nano scale size using modern VLSI technique an alternative for CMOS. QCA is transistor less architecture with electrons doped in quantum dots of QCA cell results in both positive and negative charges and on columbic interactions results in bistability that helps in binary information exchange and to perform computation. This is an advanced computing practice eliminates need of 
transistors in processing binary data. The fundamental phenomenon is utilization of coulomb's force for interactions between subatomic particles. Hence binary digits are represented as charge configurations in place of the conventional current levels representation. Coulomb interactions are responsible for device- device coupling and eliminate the need of current flow. With the help of clocked control of the device the functions like power gain, control of power dissipation as well as pipelined computation are put through. It improves integration density up to 1012 cells/cm and terahertz of switching frequency. Important notable feature is its potential to cross wires in a plane. This new information representation method called a processing in wire method. Innovative designs from QCA are

- QCA T Flipflop

- 5 Input majority gates

- Serial communication system

- $2 \mathrm{n}$ to 1 multiplexer

QCA also involves in morphological edge detection, thresholding, noise removal. Quantum Dot Cellular Automato overcomes hardware implementation difficulties in existing algorithm. Mathematical morphology algorithm for implementing complex algorithm is a field of image processing using tools in image processing applications. Mathematical morphology process involves two tasks erosion and dilation. It involves two sets of input the input and structuring element which is binary structuring element white pixel representing back ground and black pixel representing for ground. Superimpose input and structure elements. In conventional technique 0 and 1 are represented as voltage or current where in quantum dot array the information is stored as electron pairs. The QCA cell representation is denoted in Figure 5. QCA cell is a combination of four quantum dots in a square array by tunnel barrier (Interaction between the dots by coulomb interactions and Quantum mechanical tunneling). The electron tunnels across dots but not outside the cell. The two motion electrons are represented on opposite corners of a cell.

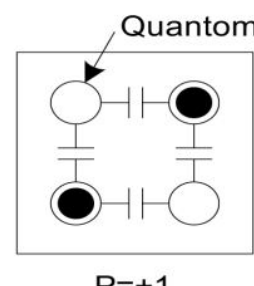

(Binary 1)

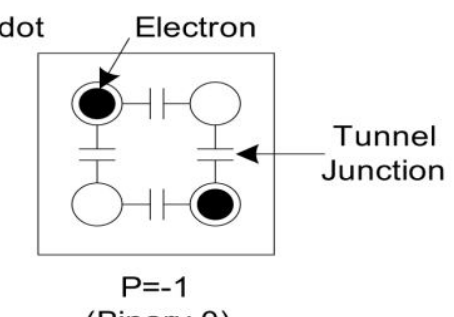

(Binary 0)
Fig. 5: QCA cell representing binary representation " 0 " and " 1 ".

QCA designs include successive placement of QCA cells that act as a binary wire, 3 input majority gates implemented using five cells. Majority gate act as a configurable AND or OR gate with 2 input $A$ and B and Sel that controls behavior. When QCA AND or OR gate connected with not gate sequential and combinational circuits can be designed. Figure 6 explains various QCA designs.

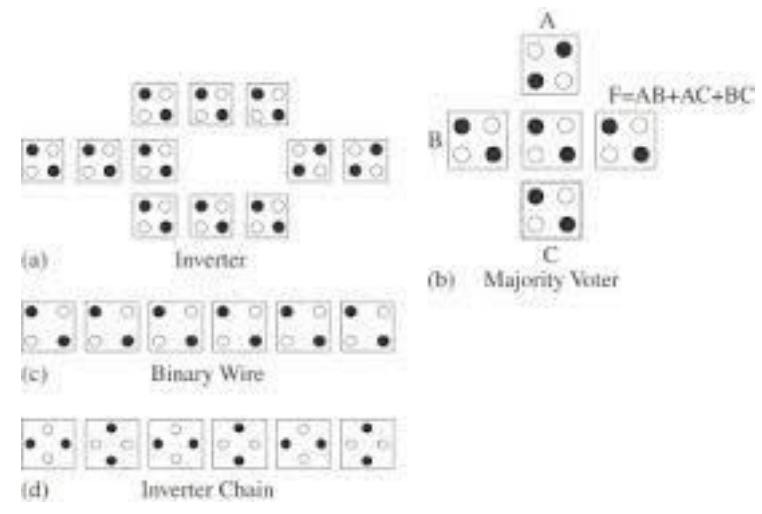

Fig.6: QCA cell designs

The majority gate with two input $\mathrm{A}$ and $\mathrm{B}$ results in logic AND output when third input Sel is 0 and logic OR when Sel value is 1 . Thus, tunnel junction act as a capacitor couple's potential on dot to other to provide path for quantum mechanical tunneling shows rearrangement of single charges with neighbor cell to perform computation task.

\section{Application Role of Quantum Dot Cellular Automato Process in Image Processing Techniques}

\subsection{Image Masking}

QCA plays an important role in image masking techniques includes designing of low power encoder circuit Stegnalysis performed to show retrieval of encoded image without employing secret key. This methodology involves conversion of input image in to binary image and key from the user called the cipher image undergoes xor operation and resulted in binary image. The encoding operation is explained in the figure 7 .

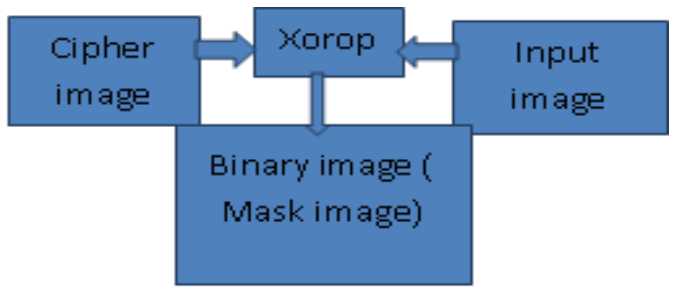

Fig.7: Encoder design resulted with mask image

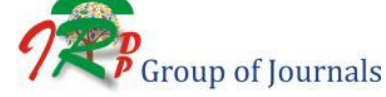


The process is divided in to four processes. The mas resulted output constituting cipher image and mask image is sent to receiver. The cipher image when undergoes xor operation with mask image results in original input image. The decoding operation is explained in the figure 8. This resulted in retrieval of original image.

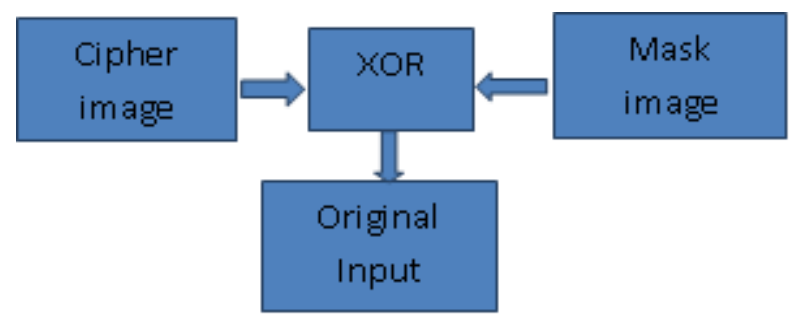

Fig.8: Retrieval of original image using decoder circuit

Morphological erosion and dilution involves the process of superimposing the structure element on image. If black pixel of the structuring element coincides with black pixel is black at the output. The Sel input decides logic gate at the output. In case of Sel input set as "0" erosion operation takes place and resulting in AND majority gate at the output. If Sel input is set to " 1 " then the dilation operation takes place and resulting in OR majority gate at the output.

\subsection{Image Enhancement}

Filtering of multichannel images play an important role in processing color images as colors conveys information about objects in scene. Degradation of signal quality due to noise, aperture correction causes destruction in image data denoted as impulsive function which occurs as a short time duration high energy spikes. Impulsive noises caused due to manmade activities or natural phenomenon including Television signal transmission through UHF and VHF. Two stage directional marginal median filters used for reduction of image noises and for lower computational complexity. The Quantum Dot Cellular Automato steps involve threshold decomposition, binary median filter and adder operation. In threshold decomposition an element $\mathrm{M}$ is converted in to $2^{\wedge} \mathrm{b}$ bit element applied to first stage binary filter designed using 5bit binary median filter. Second stage binary filter is using $\mathrm{m}$ as input and output of previous stage (The sum of output at second stage filter).

\section{Future Scope}

Image classification from given image initially requires the removal of noise which is best done by converting image to a gray scale. This gray scale image is considered as a binary image which could be subjected to various computing techniques which operate on binary data The technique of Quantum Dot Cellular Automata would be employed to the image in the gray scale or binary data format for the process of image classification.

\section{Conclusion}

This paper presents the innovation technology called Quantum Dot Cellular Automato in designing VLSI architecture which finds its application in image processing resulting in reduced power consumption and reduced mathematical complexity. Thus, this paper dealt with the study of image processing techniques and the procedures carried out in ease way by employing Quantum Dot Cellular Automato process in it.

\section{References}

[1] Cardenas-Barrera, J. L., Plataniotis, K. N.Venetsanopoulos, A. N. (2002). QCA implementation of a multichannel filter for image processing. Mathematical Problems in Engineering, 8(1), 87-99.

[2] Debnath, B., Das, J. C., De, D., \& Ghosh, T. (2016). Image masking using quantum-dot cellular automata. In Devices, Circuits and Systems (ICDCS), 2016 3rd International Conference on (pp. 231235). IEEE.

[3] Haris, K., Efstratiadis, S. N., Maglaveras, N., \& Katsaggelos, A. K. (1998). Hybrid image segmentation using watersheds and fast region merging. IEEE Transactions on Image Processing, 7(12), 1684 1699 .

[4] Lent, C. S., Isaksen, B., \& Lieberman, M. (2003). Molecular quantum-dot cellular automata. Journal of the American Chemical Society, 125(4), 1056-1063.

[5] Lent, C. S., Tougaw, P. D., Porod, W., \& Bernstein, G. H. (1993). Quantum cellular automata. Nanotechnology, 4(1), 49.

[6] Maragos, P., \& Schafer, R. (1987). Morphological filters--Part I: Their set-theoretic analysis and relations to linear shift-invariant filters. IEEE Transactions on Acoustics, Speech, and Signal Processing, 35(8), 1153-1169.

[7] Russ, J. C. (2016). The image processing handbook. CRC press.

[8] Sen, B., Anand, A. S., Adak, T., \& Sikdar, B. K. (2011). Thresholding using quantum-dot cellular automata. In Innovations in Information Technology (IIT), 2011 International Conference on (pp. 356-360). IEEE.

[9] Suganiya, P. J., \& Ferdin, J. (2014). A Novel Structure for Image Negative using Quantum Cellular Automata Adder. International Journal of Engineering Resesrch \& Technology (IJERT), 3, 84-88.

[10] Tehrani, M. A., Safaei, F., Moaiyeri, M. H., \& Navi, K. (2011). Design and implementation of multistage interconnection networks using quantum-dot cellular automata. Microelectronics Journal, 42(6), 913-922. 